\title{
ANALYSIS OF INFLUENCING FACTORS OF CURVE MATCHING BASED GEOMETRIC CALIBRATION FOR ZY3-02 ALTIMETER DATA
}

\author{
M. Zhou ${ }^{1}$, L. S. Chen ${ }^{1,2}$, J. H. Wang ${ }^{1}$, G. E. Teng ${ }^{1}$, C. R. Li ${ }^{1}$, , Q. Q. Yao ${ }^{1}$, J. Y. Chen ${ }^{1}$ \\ ${ }^{1}$ Key Laboratory of Quantitative Remote Sensing Information Technology, Academy of Opto-Electronics, Chinese Academy of \\ Sciences, Beijing 100094, China - (zhoumei, tenggeer, crli, qqyao, jychen)@aoe.ac.cn, jinhu.wang@aircas.ac.cn \\ ${ }^{2}$ University of Chinese Academy of Sciences, Beijing 100049, China - chenlinsheng16@ mails.ucas.ac.cn
}

KEY WORDS: Satellite Laser Altimeter; Geometric Calibration; Field-free Calibration; Factor Analysis

\begin{abstract}
:
High-precision on-orbit geometric calibration of spaceborne laser altimetry data is essential to its effective applications. Firstly, the existing calibration methods for laser altimeter data are analyzed. Then, a geometric calibration method based on curve matching is proposed. Compared to the existing methods, the proposed method does not rely on ground calibration field. Thus, it is efficiency in expense and time. Notably, three factors, i.e. matching method, initial control point selection and the step size of matching step, which significantly affect the results of calibration are analyzed respectively. The analysis was validated based on the original laser altimetry data obtained by ZY3-02 satellite. According to the results, the following conclusions can be drawn preliminarily: (1) Both the correlation coefficient maximum (COR) criterion and the mean square error minimum (MSD) criterion in the curve matching can be used to correct the systematic error in altimetry data. (2) The initial control points of the selected track should have a significant change trend and the slope within the laser footprints should be less than $15^{\circ}$. (3) Current experimental data show that the best step size for matching search is $10 \mathrm{~m}$. The relevant conclusions can provide reference for the research of geometrical calibration and data processing of the same type of laser altimetry satellite.
\end{abstract}

\section{INTRODUCTION}

The ZY3-02 satellite was launched in May 2016, which equipped with earth observation experimental laser altimeter and effective altimetry data was obtained (Tang,2017). Because of the vibration during launching and the change of work environment, the pointing angle and ranging calibration parameters of the altimeter were changed with respect to the ground measured value. So that there are systematic errors in the data(Yue,2014). According to the principles of the spaceborne laser altimeter, there are mainly errors such as pointing angle, ranging, attitude and orbit position during the working process of the altimeter. The orbit position error is a random error, which can be reduced by the precise orbit determination. Most of the systematic errors are included in the attitude, pointing and ranging deviations. The attitude and pointing errors have a direct impact on the plane accuracy. The ranging error will directly affect elevation accuracy. Tidal and atmospheric delays in ranging error factors can be eliminated by tidal and atmospheric delay correction models(Han,2016).

Currently, the on-orbit geometric calibration method for spaceborne laser altimeter can be categorized into natural terrain calibration method and ground detector calibration method(Yi,2016.Sirota,2005.Schutz,Martin,2005,Magruder,2 003). The natural terrain calibration method requires the satellite to perform attitude maneuver or analyze the waveform data to compare the known terrain to calculate the calibration parameters. The ZY3-02 satellite only has the ability to roll across trajectory and the attitude maneuver ability is weak. The ranging system only provides the ranging results and does not transmit the waveform data. Thus, it cannot be calibrated by using the natural terrain calibration method(Han,2016). The ground detector calibration method requires a large number of outdoor detectors to capture laser signals to determine the precise position of laser footprint control points. Then, the calibration parameters are calculated according to the difference between the laser footprint original coordinates and the control point coordinates. The accuracy and reliability of ground detector calibration method are high. However, it requires ground calibration field and time consuming and labor inefficiency. Therefore, some scholars have proposed no-field calibration method for spaceborne laser altimeter(Zhang,2017). Which means the geometric calibration of the laser altimeter is performed without ground calibration field to reduce calibration expenses. Tang et al(2016) proposed a method to estimate the pointing angle of ZY3-02 satellite altimeter based on pyramid topographic matching, which provides support for the implementation of subsequent ground detector calibration (Tang,2017). Zhang et al(2017) used the known topographic data to match the initial altimetry value to estimate the initial direction of the ZY3-02 satellite altimeter. This method is able to determine the position coordinates of laser footprints and provide a basis for establishing laser geometric calibration field.

Based on the analysis of the existing calibration methods of space-borne laser altimeter, this work presents an on-orbit geometric calibration method of altimeter based on curve matching. Notably the three factors which significantly affect calibration results were analyzed. Firstly, the proposed altimeter geometric calibration model is analyzed and deduced. Next, the curve matching based geo-calibration method is introduced. Finally, the three factors that affect the geometric matching effect of curve matching is analyzed. ZY3-02 laser altimetry 0-level data are used to verify the experiment. According to the experimental results, the applicability of the two judgment criterions of best Matching, the reference criteria of the initial control point selection and the best step size of the matching search are given. The relevant conclusions can provide reference for the research of geometrical calibration and data processing of the same type of laser altimetry satellite.

\footnotetext{
* Corresponding author : crli@aoe.ac.cn
} 


\section{METHODOLOGY}

\subsection{Geometric calibration model}

The spaceborne laser altimetry system consists of multiple subsystems, where the laser rangefinder measures the precise distance of the satellite to the ground target, the satellite sensor measures the attitude angle, and the dual-frequency GPS antenna measures the coordinates of the satellite in the WGS84 reference frame. The distance measurement obtained by laser rangefinder is converted to three-dimensional coordinates under WGS84 reference frame by combining satellite position and attitude angle. The geometric calibration model of altimeter is given by Formula (1):

$$
\left[\begin{array}{c}
X \\
Y \\
Z
\end{array}\right]_{C T S}=R_{C I S}^{C T S} R_{S E}^{C I S} R(\omega) R(\varphi) R(\kappa)\left[R_{L}^{E}\left[\begin{array}{c}
0 \\
0 \\
k_{1} \rho+k_{2}
\end{array}\right]+\left[\begin{array}{c}
X_{L}^{E} \\
Y_{L}^{E} \\
Z_{L}^{E}
\end{array}\right]\right]+\left[\begin{array}{c}
X_{S} \\
Y_{S} \\
Z_{S}
\end{array}\right]_{C T S}
$$

$\left[\begin{array}{lll}X & Y & Z\end{array}\right]_{C T S}^{T}$ is the coordinate of the laser footprint in the WGS84 reference system; $R_{C I S}^{C T S}$ is the rotation matrix of the spatial fixed inertial reference system to the fixed ground reference system of the Earth; $R_{S E}^{C I S}$ is the rotation matrix of the orbital coordinate system to the space fixed inertial reference frame; $\omega, \varphi, \kappa$ represents the attitude angle of the satellite body relative to the satellite flight orbit, which is obtained by the satellite sensor; $R_{L}^{E}$ is the rotation matrix of the laser scanning coordinate system to the body coordinate system; $\left[\begin{array}{ccc}X_{L}^{E} & Y_{L}^{E} & Z_{L}^{E}\end{array}\right]^{T}$ is the linear offset from the center of the laser scanning center to the center of the body coordinate system; $\left[\begin{array}{lll}X_{S} & Y_{S} & Z_{S}\end{array}\right]^{T}$ is the position of the satellite centroid in the WGS84 coordinate system; $\rho$ is the laser ranging value corrected by atmospheric delay and tidal error. The calibration parameters of the laser altimetry system are set to attitude angle parameters $(\omega, \varphi, \kappa)$ and laser ranging parameters $\left(\mathrm{k}_{1}, \mathrm{k}_{2}\right)$, here $\mathrm{k}_{1}$ is the ranging multiplier constant which corrects the alignment error, $\mathrm{k}_{2}$ is the ranging plus constant which corrects the fixed error. Taking the above five error corrections as unknowns, the laser geometric calibration model is linearly expanded by Taylor's formula, and the error equation is shown in Formula (2). In Formula (2), $\left[\begin{array}{lll}X & Y & Z\end{array}\right]_{C T S}^{T}$ is the real coordinate of the laser footprint in the WGS84 reference system, $\left[\begin{array}{lll}X & Y & Z\end{array}\right]^{T}$ is the measurement of the laser footprint. The error correction is calculated using the least squares original.

$$
\left[\begin{array}{c}
X \\
Y \\
Z
\end{array}\right]_{C T S}=\left[\begin{array}{c}
X_{0} \\
Y_{0} \\
Z_{0}
\end{array}\right]+\left[\begin{array}{ccccc}
\frac{\partial X_{C T S}}{\partial \omega} & \frac{\partial X_{C T S}}{\partial \varphi} & \frac{\partial X_{C T S}}{\partial \kappa} & \frac{\partial X_{C T S}}{\partial k_{1}} & \frac{\partial X_{C T S}}{\partial k_{2}} \\
\frac{\partial Y_{C T S}}{\partial \omega \omega} & \frac{\partial Y_{C T S}}{\partial \varphi} & \frac{\partial Y_{C T S}}{\partial \kappa} & \frac{\partial Y_{C T S}}{\partial k_{1}} & \frac{\partial Y_{C T S}}{\partial k_{2}} \\
\frac{\partial Z_{C T S}}{\partial \omega} & \frac{\partial Z_{C T S}}{\partial \varphi} & \frac{\partial Z_{C T S}}{\partial \kappa} & \frac{\partial Z_{C T S}}{\partial k_{1}} & \frac{\partial Z_{C T S}}{\partial k_{2}}
\end{array}\right]\left[\begin{array}{c}
\Delta \omega \\
\Delta \varphi \\
\Delta \kappa \\
\Delta k_{1} \\
\Delta k_{2}
\end{array}\right]
$$

\subsection{Geometric calibration using curve matching}

Because China's high-resolution remote sensing satellites generally is a large platform and utilize three-axis attitude stabilization system(Han,2016), during the on-orbit operation of the altimeter, the pointing of the system is relatively stable and the attitude error of the altimeter on the whole orbit merely changes. So that the actual trajectory of the laser footprint should be approximately parallel to the trajectory of the system without error and the shape of the elevation curve tends to be similar (Chen, 2018). As shown in Fig. 1 (a), the red points are footprints calculated without systematic error projected horizontally on DEM. The black points are the actual position of the laser footprints on DEM. Fig. 1 (b) is a schematic diagram of the similarity between the original laser elevation curve and the actual ground DEM elevation curve. Based on the above principles, the initial laser footprint sequence plane coordinates is used to search reference DEM to obtain the actual position of the laser footprint as control point for the calibration parameter calculation. The specific

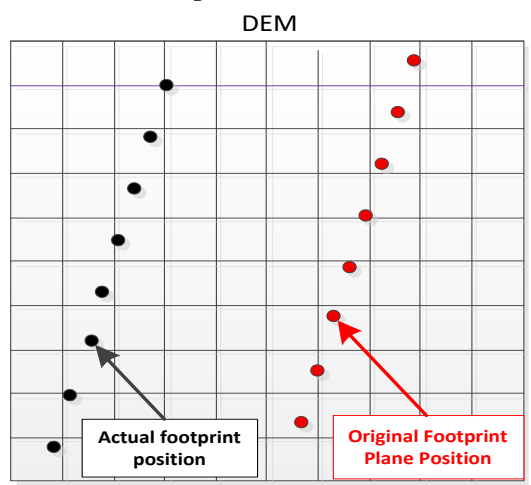

(a) Schematic diagram of laser point offset.

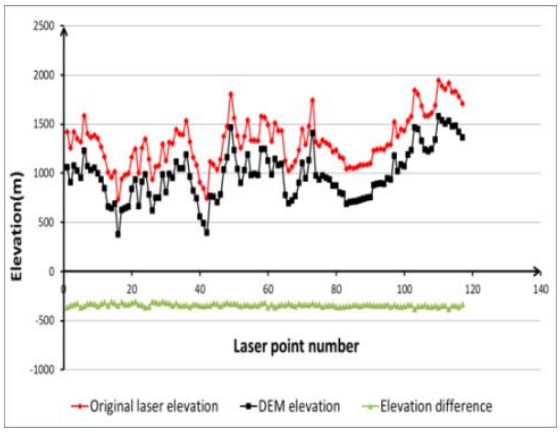

(b) Schematic diagram of elevation curve similarity. Figure 1. Schematic diagram of laser point offset and elevation curve similarity.

method is as follows:

1.Calculate the initial coordinates of the laser point, obtain point set $\mathrm{S}\left(B_{0} L_{0} H_{0}\right)$ and form the initial elevation curve curve- 0 by $\mathrm{S}\left(H_{0}\right)$;

2.The laser initial plane coordinate $\mathrm{S}\left(B_{0} L_{0}\right)$ is translated along the DEM grid with a certain step size and $\mathrm{S}(\mathrm{H})$ set is obtained by interpolation. Then, another $\mathrm{n}$ items DEM elevation curves are formed: curve- $1, \ldots$, curve-n;

3.Match the original elevation curve curve- 0 with the other $n$ DEM point elevation curves. The best matching DEM point set is used as the control point of the initial laser footprint to participate in the calculation of the calibration parameters.

The 0-level laser data contains errors such as atmospheric delay and tidal error. Thus, data preprocessing is needed, which is accurate atmospheric delay and tidal error correction model are constructed to correct the original laser ranging value. Using the corrected laser ranging data, satellite attitude and orbit data and ground control points, the geometric calibration parameters of the system can be obtained according to the least square principle. Then, the parameters are used to calculate laser footprint coordinates. The elevation values obtained by interpolation of laser footprint plane coordinates on DEM are compared with laser elevation values to evaluate the calibration effect.

\section{ANALYSIS OF INFLUENCING FACTORS}

The results of the experiments show that the calibration effect of this calibration method is closely related to the selection of 
initial control points of laser footprint and other factors. Comparing the effects of two different curve matching methods, different strategies of initial control point selection and different step size of curve matching search on the results of geometric calibration based on curve matching, a better calibration strategy is proposed to improve the effect of geometric calibration.

\subsection{Impact analysis of matching method}

At present, there are two commonly used terrain curve matching criteria: one is correlation coefficient maximum criterion (COR). The other is mean square error minimum criterion (MSD)(Chui,2011). there is not yet article to compare and evaluate the application of the COR and MSD criterion in the curve matching for geometric calibration of spaceborne laser altimetry data.

The experiment uses the two methods mentioned above as the criterion to get the best result of the curve matching. The initial data of the laser footprint control point are ZY3-02 satellite 632nd, 654th, 675th, 702nd height measurement data, the technical parameters of the altimeter are shown in Table 1. The terrain data is ASTER GDEM V2 with a spatial resolution of $1^{\prime \prime} \times 1^{\prime \prime}$ (approx. 30m x 30m) and an elevation accuracy of $20 \mathrm{~m}$. Tables 2 is the list of calibration parameters calculated according to the COR and MSD criterion.

\begin{tabular}{|c|c|}
\hline Parameters & Index \\
\hline Laser beam & 1 \\
Laser frequency $(\mathrm{Hz})$ & 2 \\
Track height $(\mathrm{km})$ & 505 \\
Laser footprint spacing $(\mathrm{m})$ & $\approx 3500$ \\
Footprint diameter $(\mathrm{m})$ & $\leq 75$ \\
Laser wavelength $(\mathrm{nm})$ & 1064 \\
Ranging accuracy $(\mathrm{m})$ & $1\left({\left.\text { slope }<15^{\circ}\right)}^{\circ}\right.$ \\
\hline
\end{tabular}

Table 1. ZY3-02 laser altimeter technical parameters.

From Table 2, it can be seen that the calibration parameters obtained from the control point data of each track are obviously different because of the difference between the offset in X-direction and Y-direction respectively. Even with the raw data of the same control point, different results are

\begin{tabular}{|c|c|c|c|c|c|c|c|c|}
\hline Criterion & Track & X offset $/ \mathrm{m}$ & Y offset $/ \mathrm{m}$ & Roll $^{\circ}$ & pitch $^{\circ}$ & yaw $^{\circ}$ & $\mathrm{k}_{1}$ & $\mathrm{k}_{2} / \mathrm{m}$ \\
\hline \multirow{4}{*}{ COR } & 632 & -536 & 85 & 0.020439 & 0.057277 & 0 & 0.996459 & 1448.406 \\
& 654 & -490 & 76 & 0.022206 & 0.051242 & 0 & 1.012491 & -6688.545 \\
& 675 & -480 & 43 & 0.016597 & 0.051849 & 0 & 0.999917 & -303.044 \\
& 702 & -457 & -19 & 0.011020 & 0.050348 & 0 & 0.996342 & 1514.930 \\
\hline \multirow{4}{*}{ MSD } & 632 & -400 & 186 & 0.028510 & 0.039932 & 0 & 1.002097 & -1415.231 \\
& 654 & -483 & 54 & 0.019603 & 0.051103 & 0 & 1.011979 & -6427.957 \\
\cline { 2 - 9 } & 675 & -481 & 43 & 0.016622 & 0.051959 & 0 & 0.999887 & -287.872 \\
& 702 & -457 & -18 & 0.011129 & 0.050320 & 0 & 0.996338 & 1516.646 \\
\hline
\end{tabular}

Table 2. Comparison of COR and MSD criterion obtained calibration parameters.

obtained by using different optimal matching discriminant principles. The offset in X-direction and Y-direction obtained from the two matching rules of track 632 nd are significantly different, thus the calculated calibration parameters are different. The position of control points obtained by COR and MSD criterion is very close in $675 t h, 702$ nd data, it can be said that the results are consistent under the spatial resolution of ASTER GDEM.

In the experiment, the $665 \mathrm{th}$ and $703 \mathrm{rd}$ original altimetry data were used to verify the influence of calibration parameters calculated by different methods on the correction effect. Table 3 is the statistic of the mean value and the RMSE of the 665 th and $703 r d$ elevation error before and after calibration. According to the data in the tables, we can initially draw the following conclusions: the COR and MSD criterion in the curve matching can be used to correct the systematic error in the altimetry data. The two methods have the same effect and no significant difference. Both methods can improve the accuracy of original altimetry data by more than $91 \%$.

\begin{tabular}{|c|c|c|c|c|c|c|c|c|c|}
\hline \multicolumn{2}{|c|}{ Validation Data } & \multicolumn{6}{c|}{665 th } & \multicolumn{4}{c|}{$703 \mathrm{rd}$} \\
\hline \multicolumn{2}{|c|}{ Parameter Track } & 632 & 654 & 675 & 702 & 632 & 654 & 675 & 702 \\
\hline \multirow{3}{*}{ MEAN } & Before & -344.65 & -344.65 & -344.65 & -344.65 & -342.60 & -342.60 & -342.60 & -342.60 \\
& COR & 3.35 & 4.08 & 0.01 & -3.47 & 3.39 & 4.20 & -0.05 & -3.56 \\
& MSD & 6.12 & 3.43 & 0.06 & -3.16 & 6.27 & 3.43 & 0.01 & -3.25 \\
\hline \multirow{3}{*}{ RMSE } & Before & 345.08 & 345.08 & 345.08 & 345.08 & 342.87 & 342.87 & 342.87 & 342.87 \\
& COR & 7.78 & 28.15 & 6.41 & 8.29 & 9.76 & 22.79 & 5.70 & 10.05 \\
& MSD & 12.33 & 27.03 & 6.38 & 8.16 & 11.28 & 21.60 & 5.71 & 9.94 \\
\hline Accuracy & COR & $97.75 \%$ & $91.84 \%$ & $98.14 \%$ & $97.60 \%$ & $97.15 \%$ & $93.35 \%$ & $98.34 \%$ & $97.07 \%$ \\
Improvement & MSD & $96.43 \%$ & $92.17 \%$ & $98.15 \%$ & $97.64 \%$ & $96.71 \%$ & $93.70 \%$ & $98.33 \%$ & $97.10 \%$ \\
\hline
\end{tabular}

Table 3. Error statistics of $665 \mathrm{th}$ and $703 \mathrm{rd}$ original altimetry data before and after calibration

\subsection{Impact analysis of initial control point selection}

It can be seen from the previous data in part 3.1 that the effect of calibration methods correlates with the initial control altimetry data. Therefore, this paper analyzes the elevation distribution of the 632nd, 654th, 675th, and 702nd track data and study the effect of the initial control point selection on the result of the calibration method. Figure 2 shows the distribution of elevation and slope within the laser footprint of the control points in each track.

According to the slope range of the laser footprints in each track initial control point, the number and proportion of each slope range are obtained, as shown in Table 4. 


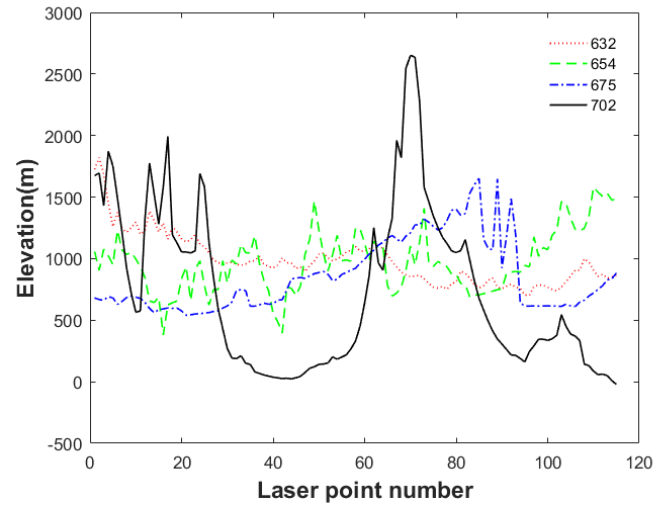

(a) Elevation distribution.

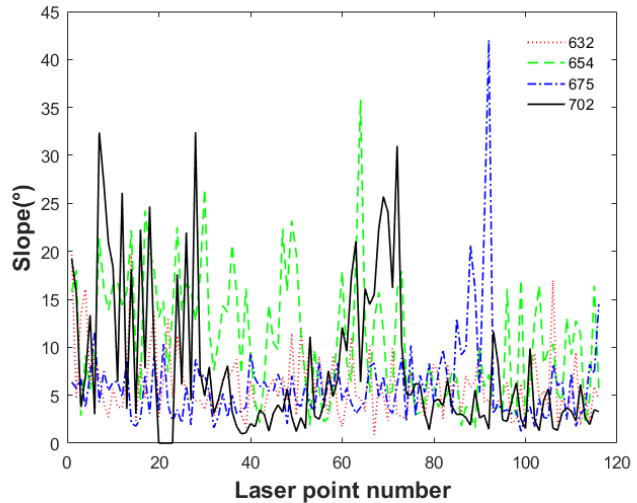

(b) Slope within the laser footprints.

Figure 2. Distribution of elevation and slope within the laser footprints.

\begin{tabular}{|c|c|c|l|c|}
\hline TracklSlope & $0-5^{\circ}$ & $5^{\circ}-15^{\circ}$ & $15^{\circ}-35^{\circ}$ & $35^{\circ}-55^{\circ}$ \\
\hline 632 & $62(52.99 \%)$ & $51(43.59 \%)$ & $4(3.42 \%)$ & $0(0 \%)$ \\
654 & $27(23.08 \%)$ & $64(54.7 \%)$ & $32(27.35 \%)$ & $1(0.85 \%)$ \\
675 & $55(47.01 \%)$ & $58(49.57 \%)$ & $3(2.56 \%)$ & $1(0.85 \%)$ \\
702 & $63(53.85 \%)$ & $32(27.35 \%)$ & $22(18.80 \%)$ & $0(0 \%)$ \\
\hline
\end{tabular}

Table 4. Statistics of Laser footprints slope.

According to Table 3, the actual calibration effectiveness of control points in each track is $675>702>632>654$. Laser altimetry data generally have high accuracy in flat areas. However, in areas with large slopes, the accuracy is slightly worse, but elevation changes can clearly reflect the characteristics of the terrain. From Figure 2 and Table 4, $96.58 \%$ of the laser footprints with slope less than $15^{\circ}$ in track 675 can ensure the high quality of height measurement data. A small part of the data distributed in area with large slope can highlight the topographic characteristics and the topography has obvious changing trend. Therefore, the combination of the data quality and topographic characteristics is beneficial to obtain more accurate coordinates of control points in curve matching. Similarly, the topographic characteristics of track 702 data are obvious. The $53.85 \%$ of the footprint slope is below $5^{\circ}$ and the overall data quality is good, which ensures the effective calibration results. The slope of the control points in track 632 is below $15^{\circ}$, which depicts excellent data quality. However, its elevation change is unobvious and cannot better represent the topographic characteristics of the data. Therefore, the lack of obvious features in curve matching fail to get more accurate control points, which makes the calibration effect slightly worse. The elevation variation between adjacent points of track 654 is very frequent and there is no general trend. $28.2 \%$ of the laser footprints have a slope of larger than $15^{\circ}$ and only $23.08 \%$ of the laser footprints have a slope of less than $5^{\circ}$. Therefore, the overall measurement accuracy is lower and the calculated calibration parameters are not well satisfied.

From the above analysis, the calibration parameters obtained by curve matching calibration method of track 675 are the best. Because the slope of more than $96 \%$ of the initial laser footprint control points is less than $15^{\circ}$, which guarantees better data quality. Although more than $96 \%$ of the initial laser footprint of 632 track has a terrain slope less than $15^{\circ}$. The terrain has no obvious changing trend compared with that of track 675 the calibration effectiveness is slightly lower. Therefore, when using curve matching calibration method, the elevation of the initial control points of the selected track should have a significant change trend and the slope within the laser footprints should be less than $15^{\circ}$. This ensures to obtain more accurate control points in curve matching, and then calculate more accurate calibration parameters.

\subsection{Impact analysis of matching search step size}

Normally, the horizontal error of the original laser altimetry is unknown and a large area is needed to search and match the real position of the laser footprint. Therefore, an appropriate matching search step size is essential to efficient matching. In this work, by analyzing the changes of calibration parameters and calibration effect with different matching search step sizes, we can determine the appropriate curve matching step size and improve the speed of matching. In the experiment, ZY3-02 satellite 675th laser footprint data is used as the initial control points and DEM data is still ASTER GDEM V2. The search step of curve matching is set to 13 grades from 1000 meters to 1 meter. The best matching result is determined by COR criterion. Table 5 shows the variation of calibration parameters with search step.

It can be seen from Table 5 that with the decrease of matching step size, the calibration parameters are gradually approaching the 1-meter parameters, and no significant changes occur when the step size is less than 10 meters. Because the X-offset and Y-offset of the laser footprint are very different between large search step size and small search step size, the resulted calibration parameters calculated differ dramatically. When the step size decreases gradually, the offset in $\mathrm{X}$ and $\mathrm{Y}$ direction tends to be consistent, so the calibration parameter tends to be stable.

The ZY3-02 satellite 665th altimetry data is used as verification data to evaluate the calibration result. In the experiment, the calibration parameters obtained with step size from 1000 meters to 1 meter are used to calibrate the original altimetry data. Table 6 is the mean and RMSE of elevation error before and after calibration with different step sizes. Fig. 3 shows the elevation error and computation time variation tendency. It can be seen from the Table 6 and Fig. 3 that the mean of elevation error has almost no change when stepping to 10 meters, and the RMS has no obvious difference when stepping to 80 meters. The time spent in matching process increases as the step decreases. The computation time of stepping to 10 meters is two-thirds of that of 1 meter, but there is no obvious difference in the accuracy of their results. 
Therefore, on the premise of ensuring the calibration effect, step for matching search is 10 . when the spatial resolution of DEM is $30 \mathrm{~m} \times 30 \mathrm{~m}$, the best

\begin{tabular}{|c|c|c|c|c|c|c|}
\hline step & Roll $\left.^{\circ}\right)$ & $\left.\operatorname{Pitch}^{\circ}\right)$ & $\mathrm{k}_{1}$ & $\mathrm{k}_{2}(\mathrm{~m})$ & $\mathrm{X}$ offset$(\mathrm{m})$ & $\mathrm{Y}$ offset$(\mathrm{m})$ \\
\hline 1000 & -0.000031 & -0.000128 & 0.996682 & 1333.91 & 0 & 0 \\
800 & 0.019764 & 0.088273 & 0.993066 & 3163.87 & -800 & 0 \\
500 & 0.012341 & 0.055123 & 0.997939 & 696.87 & -500 & 0 \\
300 & 0.040543 & 0.0256 & 0.995495 & 1935.13 & -300 & 300 \\
100 & 0.012341 & 0.055123 & 0.997939 & 696.87 & -500 & 0 \\
80 & 0.011846 & 0.052913 & 0.999176 & 70.49 & -480 & 0 \\
50 & 0.017866 & 0.053885 & 0.999262 & 26.54 & -500 & 50 \\
30 & 0.015161 & 0.05217 & 0.999236 & 40.21 & -480 & 30 \\
10 & 0.016266 & 0.051923 & 0.999749 & -219.87 & -480 & 40 \\
8 & 0.016266 & 0.051923 & 0.999749 & -219.87 & -480 & 40 \\
5 & 0.016818 & 0.051799 & 0.999796 & -243.50 & -480 & 45 \\
3 & 0.016487 & 0.051873 & 0.999787 & -238.95 & -480 & 42 \\
1 & 0.016597 & 0.051849 & 0.999792 & -241.63 & -480 & 43 \\
\hline
\end{tabular}

Table 5. Comparison of calibration parameters at different step size.

\begin{tabular}{|c|c|c|c|c|c|c|c|}
\hline Step size & 1000 & 800 & 500 & 300 & 100 & 80 & 50 \\
\hline Mean of residual before calibration & -344.65 & -344.65 & -344.65 & -344.65 & -344.65 & -344.65 & -344.65 \\
Mean of residual after calibration & 9.85 & 20.43 & 5.40 & 13.01 & 5.40 & 1.70 & 1.61 \\
RMSE of residual before calibration & 345.08 & 345.08 & 345.08 & 345.08 & 345.08 & 345.08 & 345.08 \\
RMSE of residual after calibration & 12.40 & 22.08 & 8.35 & 14.65 & 8.34 & 6.66 & 6.37 \\
\hline Step size & 30 & 10 & 8 & 5 & 3 & 1 & - \\
\hline Mean of residual before calibration & -344.65 & -344.65 & -344.65 & -344.65 & -344.65 & -344.65 & - \\
Mean of residual after calibration & 1.23 & -0.05 & -0.05 & -0.40 & -0.34 & -0.22 & - \\
RMSE of residual before calibration & 345.08 & 345.08 & 345.08 & 345.08 & 345.08 & 345.08 & - \\
RMSE of residual after calibration & 6.49 & 6.33 & 6.33 & 6.28 & 6.32 & 6.30 & - \\
\hline
\end{tabular}

Table 6. Step size change and calibration effect analysis.

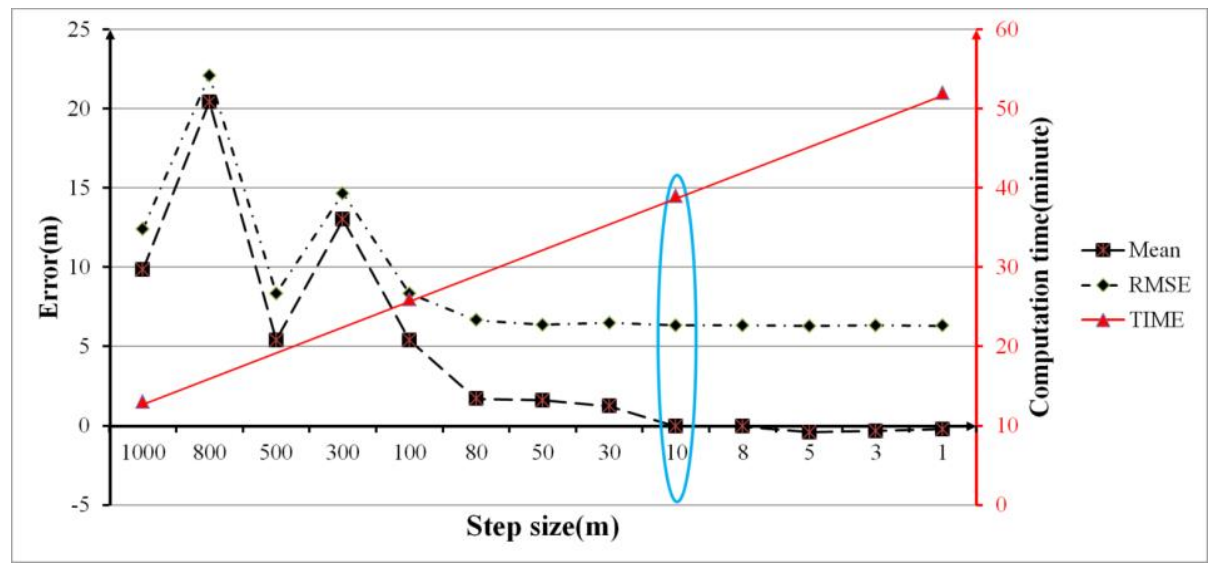

Figure 3. The elevation error and computation time change trend map.

\section{CONCLUSIONS}

By analyzing and comparing the impacts of different curve matching methods, different initial control points and different search step sizes on the results of curve matching geometric calibration, the following conclusions can be drawn preliminarily: (1) The COR and MSD criterion in the curve matching can be used to correct the systematic error in altimetry data. Those two methods have the similar effect and no significant difference. Both methods can improve the accuracy of original altimetry data by more than $91 \%$. (2)
The elevation of the initial control points of the selected track should have a significant change trend and the slope within the laser footprints should be less than $15^{\circ}$ when apply curve matching calibration method. This results in more accurate control points and better calibration parameter calculation results. (3) When the spatial resolution of DEM is $30 \mathrm{~m} \times 30$ $\mathrm{m}$, the best step size for matching search is $10 \mathrm{~m}$. We hope more accurate DEM data can be obtained to verify and further analyze the influencing factors for curve matching based geometric calibration method. 


\section{ACKNOWLEDGMENTS}

The work is supported by the [The Science and Technology Innovation Project of the Chinese Academy of Science] under Grant [No. Y70X23A34Y].

\section{REFERENCES}

Chen, L.S., Zhou, M., Teng, G.E., 2018. Non-field in-orbit geometric calibration method for spaceborne laser altimeter based on curve matching. Journal of University of Chinese Academy of Sciences, IN PRESS.

Chui, P.Y., Feng, J.H., Zhu, S.Y., 2011. 3D Terrain Feature Matching Based Navigation for Lunar Soft Landing. Journal of Astronautics | J Astrona, 32(03), 470-476.

Han, L., Tian, S.Q., Xie, J.F., 2016. Development Status of Calibration Technique for Space-borne Laser Altimeter. Spacecraft Recovery\&Remote Sensing, 37(06), 11-19.

Martin, C.F., Thomas, R.H., Krabill, W.B., 2005. ICESat range and mounting bias estimation overprecisely-surveyed terrain. Geophysical Research Letters, 32(L21S07), 1-4.

Magruder, L.A., Suleman, M.A., Schutz, B.E., 2003. ICESat laser altimeter measurement time validation system. Measurement Science And Technology, 14(11), 1978-1985.

Magruder, L.A., Webb, C.E., Urban, T.J., 2001. Pointing angle and timing calibration/validation of the geoscience laser altimeter with a ground-based detection system. IEEE-International Geoscience \& Remote Sensing Symposium, 1584-1587.

Sirota, J. M., Bae, S., Millar, P., 2005. The transmitter pointing determination in the GeoscienceLaser Altimeter System. Geophysical Research Letters, 32(L22S11), 1-4.

Schutz, B. E., Zwally, H.J., Shuman, C.A., 2005. Overview of the ICESat Mission. Geophysical Research Letters,32 (L21S01), 1-4.

Tang, X.M., Xie, J.F., Fu, X.K., 2017. ZY3-02 Laser Altimeter On-orbit Geometrical Calibration and Test. Acta Geodaetica et Cartographica Sinica, 46(06), 714-723.

Tang, X.M., Xie, J.F., Mo, F., 2017. Footprint Location Prediction Method of ZY3-02 Altimeter. Acta Geodaeticaet Cartographica Sinica, 46(07), 866-873.

Yue, C.Y., He, H.Y., Bao, Y.F., 2014. Study on Error Propagation of Space-borne Laser Altimeter Geometric Positioning. Spacecraft Recovery \& Remote Sensing,35 (02), 81-86.

Yi, H., Li, S., Weng, Y.K., 2016. On-orbit calibration of spaceborne laser altimeter using natural surface range residuals. Journal of Huazhong University of Science and Technology (Natural Science Edition), 44(08), 58-61.

Zhang, G., Li, S.N., Huang, W.C., 2017. Geometric Calibration and Validation of ZY3-02 Satellite Laser Altimeter System. Geomatics and Information Science of Wuhan University, 42(11), 1589-1596. 